\title{
Comparison of minimally invasive approaches and standard median parapatellar approach for total knee arthroplasty: A systematic review and network meta-analysis of randomized controlled trials
}

\author{
Li Zhang $^{\mathrm{a}, 1}$, Xian Li ${ }^{\mathrm{b}, 1}$, Julian M. Rüwald ${ }^{\mathrm{a}}$, Kristian Welle ${ }^{\mathrm{a}}$, Frank A. Schildberg ${ }^{\mathrm{a}, 1, *}$ and \\ Koroush Kabir ${ }^{\mathrm{a}, 1, *}$

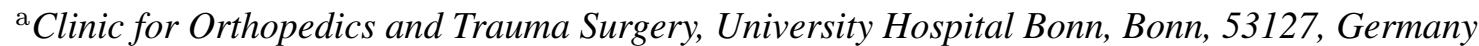 \\ ${ }^{\mathrm{b}}$ Department of Orthopedic and Trauma Surgery, Xiyuan Hospital, China Academy of Chinese Medical \\ Science, Beijing 100091, China
}

Received 1 December 2019

Accepted 18 August 2020

\begin{abstract}
.
BACKGROUND: Minimally invasive total knee arthroplasty (TKA) has been actively advertised by the orthopedic industry. The purpose of this network meta-analysis was to comprehensively compare the effectiveness of four minimally invasive surgery (MIS) approaches and the medial parapatellar (MPP) approach to improve the American Knee Society Score (KSS) in primary TKA.

MATERIALS AND METHODS: Studies were comprehensively searched on PubMed, Embase, Cochrane Library (CENTRAL), Web of Science and Science Direct up to June 2018 with a major focus on the outcome of KSS. Risk of bias was assessed using the Cochrane risk of bias tool. Quality assessment was performed using the GRADE system. Both pair-wise and network meta-analyses are calculated to comprehensively compare the effectiveness of four MIS and TKA approaches.

RESULTS: Eleven trials with 1025 knees undergoing TKA were included. Our analysis showed that both MPP and MIS approaches provided improvement in terms of short-term (four-eight weeks) total, objective and functional KSS. The network-meta analysis revealed that MIS approaches showed a trend towards superior KSS improvement over standard MPP approaches. However, statistical advantages were only observed in the mini-MPP group for functional KSS compared to the conventional MPP and quadriceps-sparing (QS) groups.

CONCLUSIONS: Evidence shows that MIS TKA approaches are effective alternatives to MPP approaches. However, orthopedic surgeons should be cautiously optimistic about minimally invasive TKA in terms of KSS improvement.

Keywords: Minimally invasive surgery, total knee arthroplasty, total knee replacement, network meta-analysis, bayesian model

\footnotetext{
${ }^{1}$ Authors contributed equally to this work.

${ }^{*}$ Corresponding author: Frank A. Schildberg and Koroush Kabir, Clinic for Orthopedics and Trauma Surgery, University Hospital Bonn, Venusberg-Campus 1, Bonn, 53127, Germany. Tel.: +49 22828719893; E-mail: frank.schildberg@ukbonn.de and koroush.kabir@ukbonn.de.
} 


\section{Introduction}

Total knee arthroplasty (TKA) is considered to be the optimal surgical method to treat advanced osteoarthritis for excellent alleviation of pain, restoration of joint function and improvement in life quality. As the most popular surgical approach in primary TKA, the medial parapatellar (MPP) approach offers excellent operative visualization at the sacrifice of peripatellar blood supply, quadriceps tendon and medial portion of the quadriceps muscle. This might lead to avascular necrosis, anterior knee pain as well as extensor mechanism weakening and thus functional outcomes remain a concern.

Minimally invasive surgery (MIS) has been promoted for quicker rehabilitation and shorter hospitalization. Until now, four MIS approaches have been well documented, including mini-medial parapatellar (mini-MPP), mini-midvastus (mini-MV), mini-subvastus (mini-SV) and quadriceps-sparing (QS). Although technically demanding, proponents of MIS surgery state improved cosmetic appeal, decreased postoperative pain, accelerated recovery as measured by quadriceps function, better early range of motion (ROM), and better cost savings as advantages in comparison to the standard TKA approach [1-4]. Possible problems with the surgical technique include its steep surgical learning curve, longer operative time, compromised surgical exposure, technical errors (e.g. fracture or component malposition), and neurovascular damage [5-11]. Several authors expressed concerns that MIS approaches may lead to component misalignment [12] and perioperative complications such as local wound-related problems, deep venous thrombosis (DVT) and pulmonary embolism (PE) [13-15].

Recent studies revealed that the MPP approach has comparative clinical results as MIS approaches. Recently, comparative studies have migrated from MIS procedures versus MPP procedure to comparisons between MIS approaches and thus more well-designed randomized controlled trials (RCTs) have been published. Therefore, we conducted a Bayesian network meta-analysis to compare the effectiveness of four MIS approaches as well as the standard MPP approach for the improvement of short-term (four-eight weeks) total, objective and functional Knee Society Score (KSS).

\section{Materials and methods}

\subsection{Inclusion and exclusion criteria}

For our analysis, we included properly designed RCTs evaluating the effects of minimally invasive approaches for TKA. To guarantee the quality of this review, we adopted a rigorous standard for the included studies. We required that the included studies adequately describe the method of randomization. Non-RCTs, abstract-only papers, and RCT protocols were excluded. We included RCT studies that described adult participants (age $\geqslant 18$ years) undergoing TKA, special subjects such as revision surgery were excluded. Case reports, cohort studies, quasi-RCTs and non-RCTs were also excluded. There was no restriction on gender or race. Five approaches: mini-medial parapatellar approach and four kinds of minimally invasive approaches were included. We required that each study should include at least one approach. It should be noted that navigation-assisted approaches were not included. The comparators included five TKA approaches. The primary outcome measure for this study was the change in total, objective and functional KSS at four-eight weeks compared to baseline. If data from more than one follow-up result were available in one period, the time points nearest to six weeks were used for the three different KSS.

\subsection{Search strategy}

PubMed, Embase, Cochrane Library (CENTRAL), Web of Science and Science Direct were searched up 
to June 2018. The following keywords were used to search the databases: arthroplasty, replacement, knee, knee prosthesis, knee replacement arthroplasty, total knee arthroplasty, total knee, TKA, mini-MV, minimidvastus, mini-subvastus, mini-medial parapatellar, quadriceps-sparing, minimally invasive surgical procedures, minimal access surgical procedures, minimal surgical procedure with the limitation of RCTs (Appendix 1). The Preferred Reporting Items for Systematic Review and Meta-Analyses (PRISMA) [16] methodology guidance was employed. To avoid the potential omission of studies, we searched additional databases for gray literature. In addition, to guarantee literature saturation, we also scanned the relevant trials enrolled by previous systematic reviews and meta-analyses related to TKA. We applied no language restrictions to our database searches.

\subsection{Study selection}

Two independent reviewers (LZ and XL) independently reviewed the titles and abstracts of all the literature retrieved by the search. Duplications were removed using Endnote X7 (Thomson Reuters Co., New York, USA). Full-text articles were obtained and examined if necessary. Then, the reviewers followed the eligibility criteria to select potentially relevant studies. If a disagreement occurred regarding the inclusion or exclusion of a study, then a third reviewer was consulted.

\subsection{Data extraction and quality assessment}

Two reviewers (LZ and XL) independently extracted the data using a pre-designed extraction form, consisting of surgical approach, components description, follow-up duration, male/female patient rate, average years, BMI and outcome measures. Next, the data were integrated. Discrepancies between the results were largely resolved through discussions; however, a third reviewer was consulted if an agreement could not be reached. The outcomes were change in total KSS, functional KSS and objective KSS scores.

Two reviewers (LZ and XL) independently evaluated the quality of the trials. The Cochrane Collaboration tool (Review Manager, V.5.2; Revman, Copenhagen, Denmark: The Nordic Cochrane Centre, The Cochrane Collaboration) was used to evaluate the risk of bias in the included RCTs. This tool covers the following domains: random sequence generation, allocation concealment, blinding, incomplete outcome data, selective reporting, and other bias.

After all outcomes were evaluated and a table summarizing the findings was created using the GRADE system [17], following the four-step approach to rate the quality of evidence in each of the direct and mixed estimates based on methods developed by the GRADE working group [18,19].

\subsection{Statistical analysis}

First, a pair-wise meta-analysis was performed using random effects models. Every pair of studies with the same treatments was calculated. Next, the results were reported as the mean difference (MD) with a corresponding $95 \%$ confidence interval (CI), in addition to the number of pairs of studies. These statistical analyses were performed using STATA with the metan package (version 14.0; Stata Corporation, College Station, Texas, USA).

Second, random effects network models were developed within a Bayesian framework using the Markov Chain Monte Carlo (MCMC) algorithm in WinBUGS (Bayesian inference Using Gibbs Sampling for Windows, version 1.4.3; Imperial College and MRC, UK) [20]. The model was based on Markov chains for 100,000 iterations after a burn-in of 50,000. A thinning interval of 10 was applied, which meant collecting one sample every 10 iterations. Consequently, 30,000 samples were obtained for each parameter. In this process, the Brooks-Gelman-Rubin method was used to assess the convergence between 
direct and indirect variances [21]. According to the theory of Brooks and Gelman, if the result of the Potential Scale Reduction Factor (PSRF) is close to or equal to 1, convergence has been reached. This result was also presented by MD, with a $95 \% \mathrm{CI}$. If the null value was not included in the $95 \% \mathrm{CI}$ of the MD, a statistically significant difference was indicated. The rank probability for each treatment was estimated by WinBUGS, and the data were then imported into STATA. Next, plots of the surface under the cumulative ranking curves (SUCRA) were generated [22]. The value of the SUCRA was presented as the percentage of the area under the curve: $100 \%$ indicates the best treatment, and $0 \%$ indicates the worst treatment. Placebo compared with other treatments was always considered and were thus presented. The network order in STATA was used to plot the MDs and 95\% CIs in these three different follow-up durations.

\subsection{Inconsistency analysis}

If there was a 'loop' (e.g., A-B-C) in the network, each comparison in the loop (e.g., A-B) might have an indirect result from the other comparisons (e.g., A-C and C-B); consequently, the direct and indirect result may be different. The inconsistency of the model was assessed by the node-splitting method [23]. If the p-value was smaller than 0.05 , an inconsistency was considered to be detected. The node-splitting models were generated by the gemtc package (version 0.6-1, http://cran.r-project.org/package=gemtc) of the R statistical software (version 3.2.3, http://www.r-project.org) [24].

\subsection{Sensitivity analysis and meta-regression}

For testing the influence of low-quality studies, a sensitivity analysis was conducted after excluding them. We recalculated the network result with the rank probability. If no significant difference occurred, the outcome of the NMA was considered valid.

Furthermore, meta-regression was used to test the relationship between the sample size and treatment effect. As recommended by the UK's National Institute for Health and Care Excellence, a single interaction term was used as the covariate [25]. The deviance information criterion (DIC) [26] was used as the measurement of model fit. Thus, a lower DIC value suggested a more parsimonious model. If the reduction of DIC was less than 3, the covariate was not considered to be associated with the result. Additionally, a regression parameter named coefficient was calculated. If the null was in the $95 \% \mathrm{CI}$ of the parameter, the association was not supported. Graphs were prepared using Sigmaplot 10.0.

\section{Results}

\subsection{Eligible studies}

Our search strategy yielded 856 potentially relevant articles. After carefully screening the titles and abstracts, 56 potentially eligible articles were obtained. After a careful full-text screening, 45 articles were discarded for the reasons listed in Fig. 1. Eleven RCTs met the inclusion criteria.

\subsection{Risk of bias and quality assessment}

The quantification of the risk of bias for the included RCTs were generated by Revman and presented in Fig. 2 All of these studies were described as "randomized". The generation of a random sequence was detailed in six reports and the allocations were only properly concealed in four of the articles. In addition, the detection bias was unclear, because there was insufficient information about the methods used to conceal the allocation in 10 of the studies. 


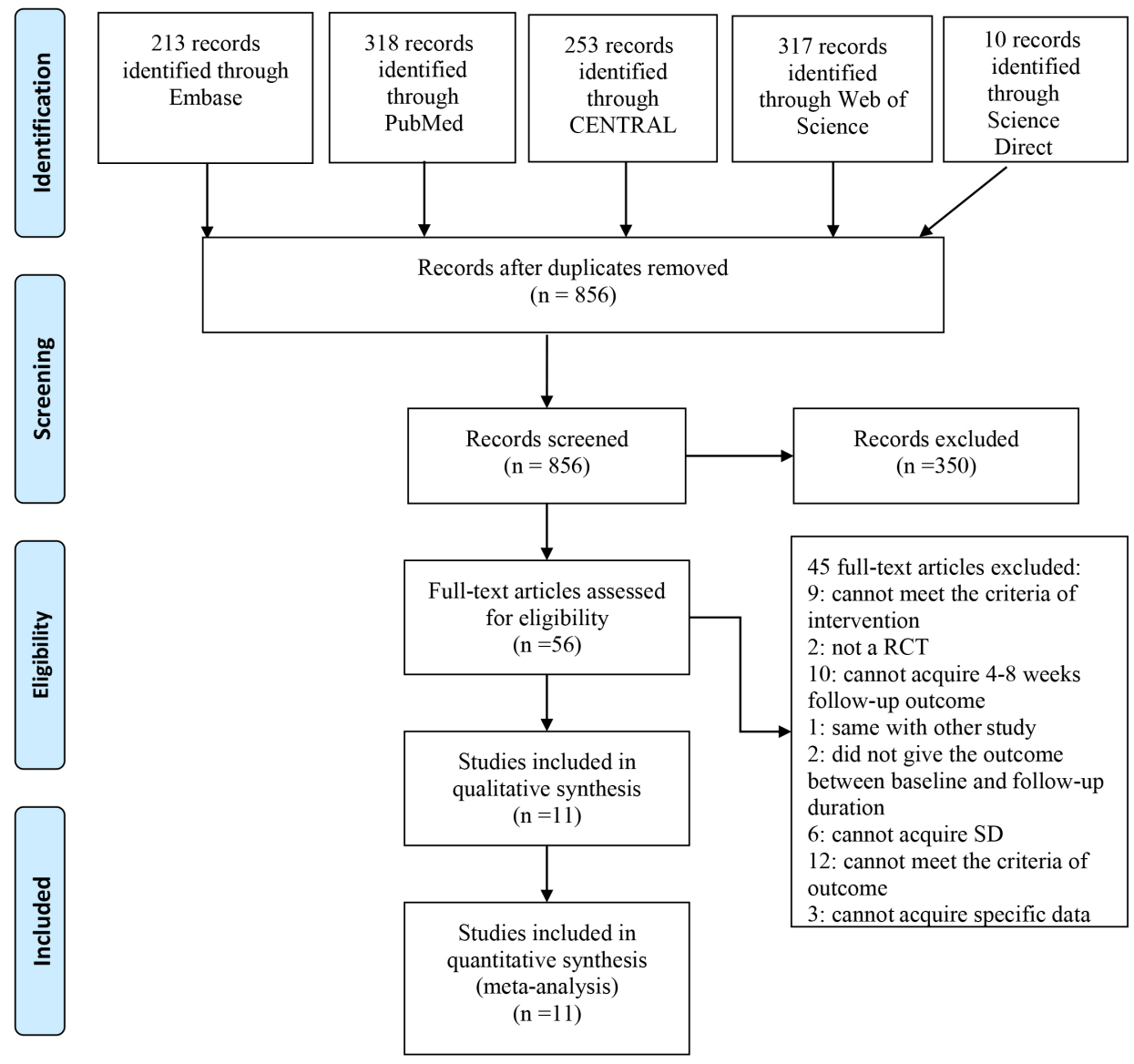

Fig. 1. PRISMA 2009 flow diagram.

\subsection{Characteristics of the included studies}

The characteristics of the included trials are presented in Table 1. Eleven trials with 1025 knees were included. The network for total, objective and function KSS included six, nine, and eight studies, respectively. The total numbers of participants in these studies were 385895 , and 761, respectively.

\subsection{Estimates of effects and quality ratings}

The results of the GRADE evaluation of interventions are presented in Appendix 2. All the reasons for downgrading are labeled. Because the design of the inclusion criteria was rigid, there was no obvious nontransitivity. Due to the inconsistency and imprecision, the confidence of the evidence of total, objective and function KSS results was low or very low.

\subsection{Network of comparisons}

Three comprehensive network plots were built using STATA (Fig. 3). The size of the circle represents the number of participants, and the thickness of the edge represents the number of studies. 


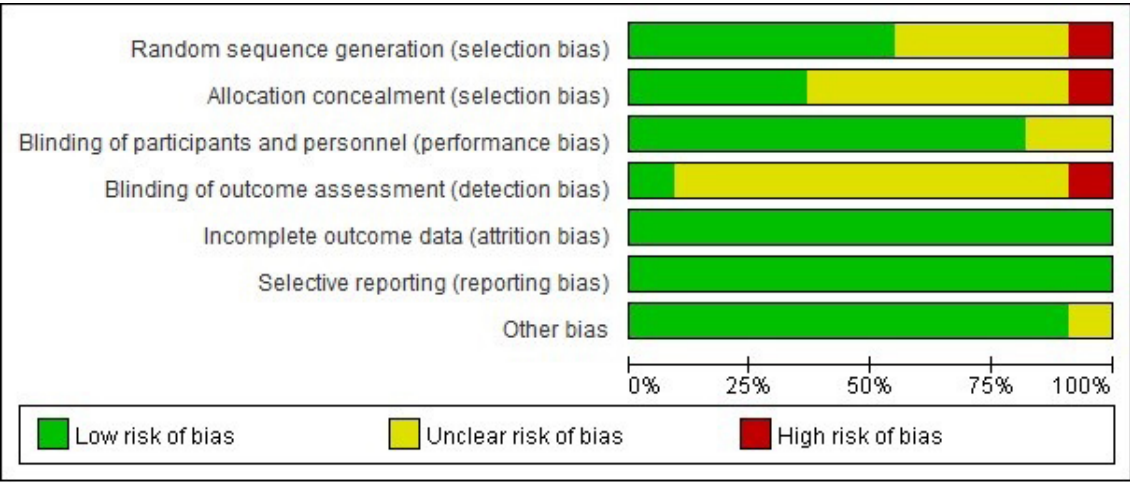

\begin{tabular}{|c|c|c|c|c|c|c|c|c|c|c|c|}
\hline 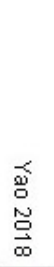 & 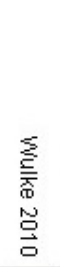 & 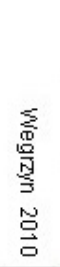 & 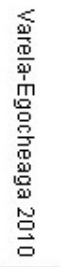 & 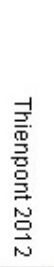 & 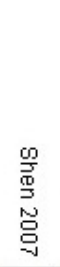 & 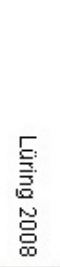 & $\begin{array}{l}\bar{\Xi} \\
\stackrel{\Xi}{\Xi} \\
\omega\end{array}$ & 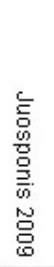 & 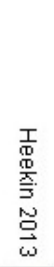 & 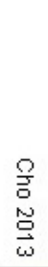 & \\
\hline$\odot$ & $\bullet$ & $\odot$ & $\odot$ & 0 & 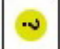 & + & + & $\odot$ & $\sim$ & $\sim$ & Random sequence generation (selection bias) \\
\hline$\odot$ & $\sim$ & $\odot$ & $\sim$ & O & $\sim$ & $\odot$ & $\odot$ & $\odot$ & $\odot$ & $\sim$ & Allocation concealment (selection bias) \\
\hline$\sim$ & + & + & + & + & $\odot$ & + & + & $\odot$ & $\odot$ & $\bullet$ & Blinding of participants and personnel (performance bias) \\
\hline$\sim$ & $\sim$ & + & 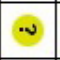 & $\cdot$ & $\sim$ & $\sim$ & $\sim$ & O & $\sim$ & $\sim$ & Blinding of outcome assessment (detection bias) \\
\hline$\odot$ & + & $\odot$ & $\odot$ & + & $\odot$ & + & + & $\odot$ & $\odot$ & $\odot$ & Incomplete outcome data (attrition bias) \\
\hline$\odot$ & + & $\odot$ & $\odot$ & + & + & + & + & $\odot$ & $\odot$ & $\odot$ & Selective reporting (reporting bias) \\
\hline$\odot$ & + & + & + & $\sim$ & $\odot$ & + & + & + & $\odot$ & + & Other bias \\
\hline
\end{tabular}

Fig. 2. Risk of bias graph (upper) and summary (lower).
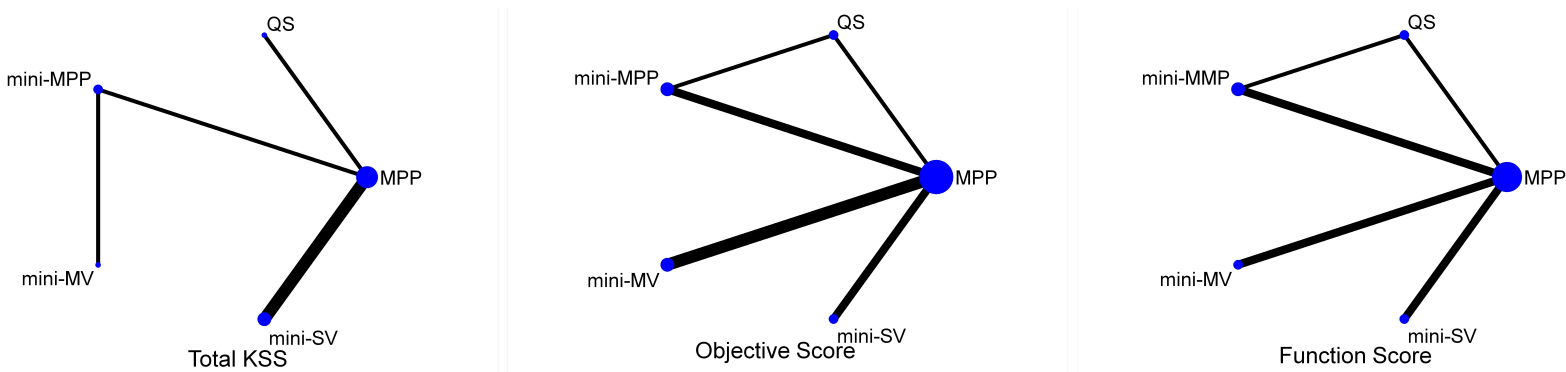

Fig. 3. Network for the comparison of the five approaches. MPP, medial parapatellar; MV, midvastus; SV, subvastus; QS, quadriceps-sparing.

\subsection{Results of the pair-wise meta-analysis}

All data were imported into STATA, and direct comparisons were made using metan with a randomeffects model. The MDs and 95\% CIs were calculated. The results are listed in the lower-left triangle of Table 2 (A, B, C, respectively) and Appendix 3. 


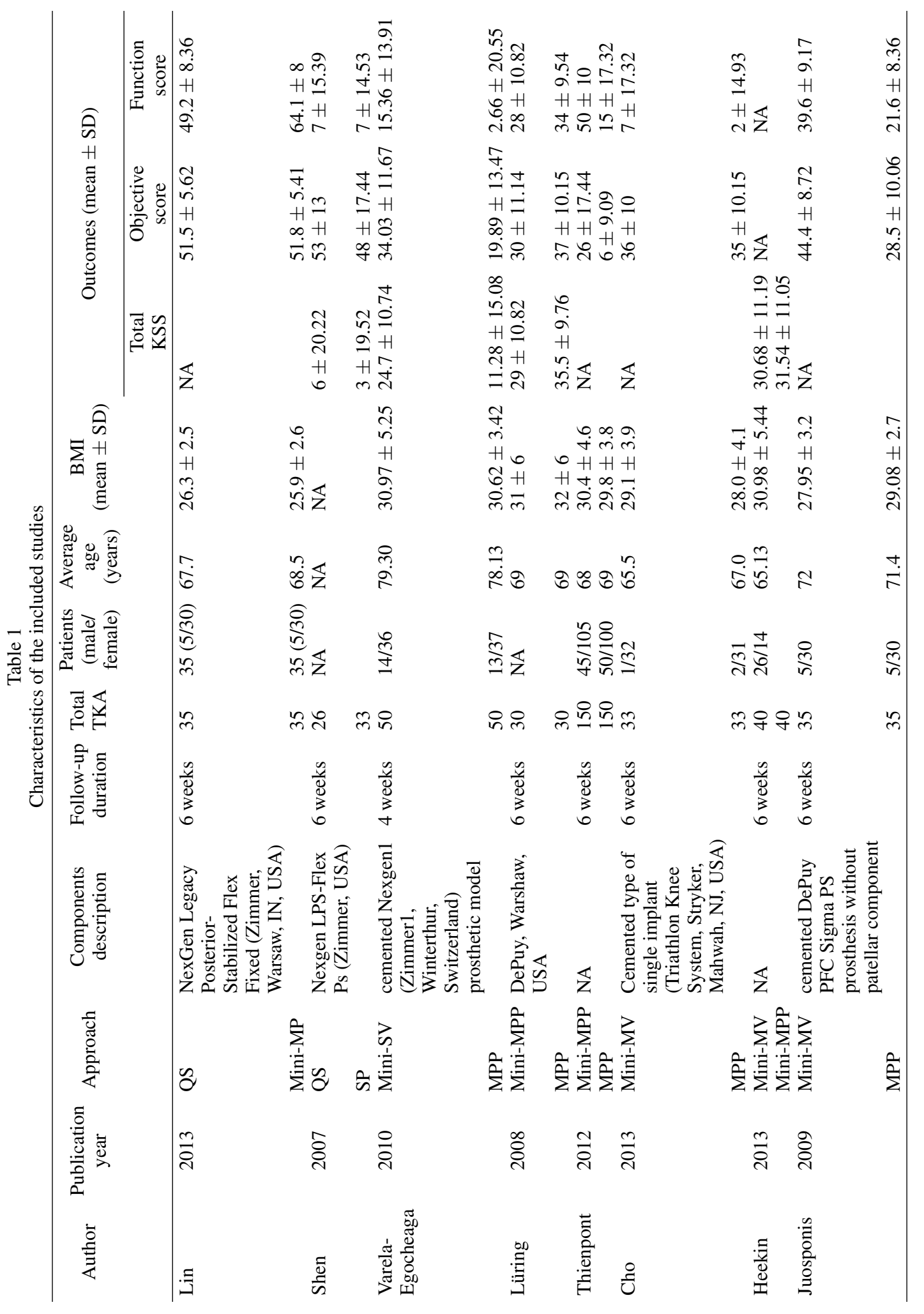




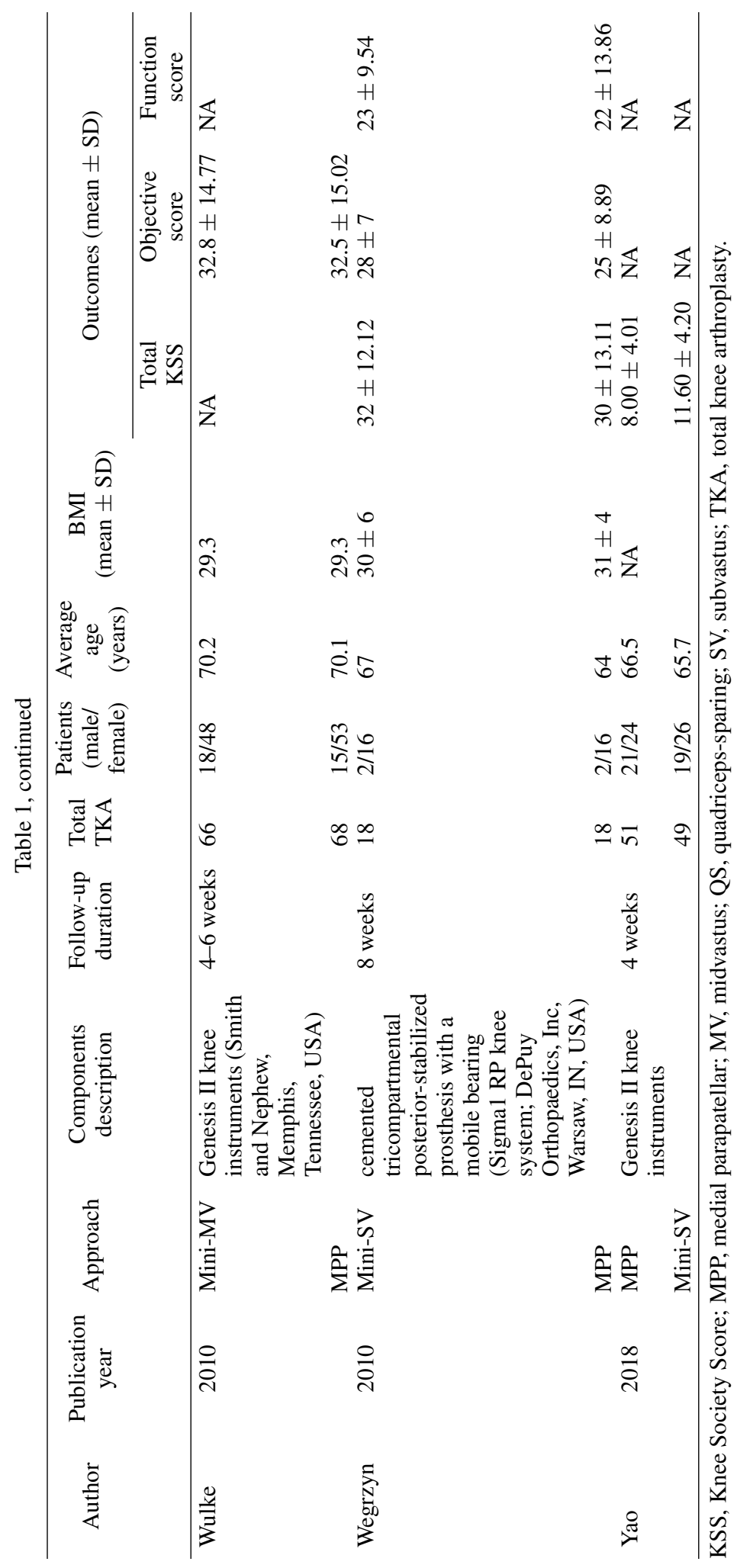


Table 2

Results of the pair-wise and network meta-analysis (mean difference, with 95\% confidence interval)

\begin{tabular}{|c|c|c|c|c|c|}
\hline $\mathbf{A}$ & 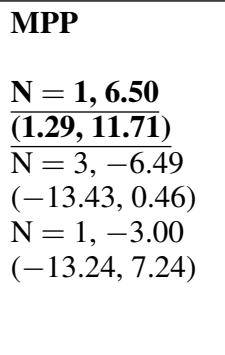 & $\begin{array}{l}6.51 \\
(-5.46,18.70) \\
\text { mini-MPP }\end{array}$ & $\begin{array}{l}-6.35 \\
(-13.39,0.67) \\
-12.86 \\
(-26.97,1.026) \\
\text { mini-SV }\end{array}$ & $\begin{array}{l}-2.95 \\
(-17.92,12.21) \\
-9.45 \\
(-28.87,9.80) \\
3.41 \\
(-13.03,20.1) \\
\text { QS }\end{array}$ & $\begin{array}{l}7.35 \\
(-9.52,24.35) \\
0.85 \\
(-11.16,12.78) \\
13.71 \\
(-4.74,32.12) \\
10.30 \\
(-12.47,32.99) \\
\text { mini-MV }\end{array}$ \\
\hline B & $\begin{array}{l}\text { MPP } \\
\mathrm{N}=2,-6.59 \\
(-33.05,19.87) \\
\mathrm{N}=2,-8.60 \\
(-19.52,2.32) \\
\mathrm{N}=1,-5.00 \\
(-12.77,2.77) \\
\mathrm{N}=3,-5.79 \\
(-16.11,4.54)\end{array}$ & $\begin{array}{l}-6.63 \\
(-17.59,4.47) \\
\text { mini-MPP }\end{array}$ & $\begin{array}{l}-8.56 \\
(-20.89,3.83) \\
-1.93 \\
(-18.59,14.48) \\
\text { mini-SV }\end{array}$ & $\begin{array}{l}-5.75 \\
(-19.56,8.15) \\
0.88 \\
(-12.61,14.19) \\
2.81 \\
(-15.68,21.42) \\
\text { QS }\end{array}$ & $\begin{array}{l}-5.73 \\
(-15.86,4.23) \\
0.90 \\
(-14.17,15.70) \\
2.83 \\
(-13.08,18.91) \\
0.02 \\
(-17.07,17.09) \\
\text { mini-MV }\end{array}$ \\
\hline $\mathbf{C}$ & $\begin{array}{l}\text { MPP } \\
\begin{array}{l}\mathrm{N}=2,-14.55 \\
(-54.73,25.63) \\
\mathrm{N}=2,-6.98 \\
(-18.45,4.48) \\
\mathrm{N}=1,0.00 \\
(-7.72,7.72) \\
\mathrm{N}=2,-11.940 \\
(-24.65,0.77)\end{array}\end{array}$ & $\frac{\frac{-15.05}{(-26.69,-3.45)}}{\text { mini-MPP }}$ & $\begin{array}{l}-6.89 \\
(-19.90,6.15) \\
8.16 \\
(-9.14,25.66) \\
\text { mini-SV }\end{array}$ & $\begin{array}{l}-0.14 \\
(-14.57,14.52) \\
\frac{\mathbf{1 4 . 9 1}}{(\mathbf{0 . 8 6}, \mathbf{2 9 . 0 4})} \\
6.75 \\
(-12.79,26.35) \\
\mathbf{Q S}\end{array}$ & $\begin{array}{l}-11.50 \\
(-24.47,1.40) \\
3.55 \\
(-13.84,21.07) \\
-4.61 \\
(-23.09,13.70) \\
-11.36 \\
(-30.96,8.31) \\
\text { mini-MV }\end{array}$ \\
\hline
\end{tabular}

a. Upper-right triangle presents the findings (MDs with 95\% CI) of the network meta-analysis conducted using WinBUGS 1.4.3. b. Lower-left triangle presents the findings (MDs with 95\% CrI) of the pairwise meta-analyses conducted using STATA 14 and N refers to the numbers of RCTs which compared the two interventions directly. c. A positive MD favors the lower-right intervention, a negative MD favours the upper-left intervention. d. Statistically significant findings are presented as bold and underlined values. MPP, medial parapatellar; MV, midvastus; QS, quadriceps-sparing; SV, subvastus.

In terms of total KSS score, mini-SV and QS conferred greater (but not significant) improvement than the standard MPP approach at four to eight weeks (MD $=-6.49,95 \% \mathrm{CI}:(-13.43,0.43)$; $\mathrm{MD}=-3.00$, 95\% CI: (-13.24, 7.24); respectively). In contrast, mini-MPP approach is associated with a significant less total KSS improvement $(\mathrm{MD}=6.50,95 \% \mathrm{CI}:(1.29,11.71))$.

In terms of objective and function score, all mini approaches showed better (but not significant) improvement than standard MPP. Notably, QS showed an equal improvement as standard MPP approach for mini-MPP. Moreover, although mini-MPP did not show a significantly better improvement compared with QS in objective score, it demonstrated significantly greater improvement ( $\mathrm{MD}=14.90,95 \% \mathrm{CI}$ : $(11.07,18.73))$ in functional score.

\subsection{Results of the network meta-analysis}

All potential comparisons were calculated via WinBUGS and are presented as MDs and 95\% CIs. All the PSRF parameters were approximately 1, indicating a strong convergence. The results are listed in the upper-right triangle of Table 2, and the significant differences are presented as bold and underlined 

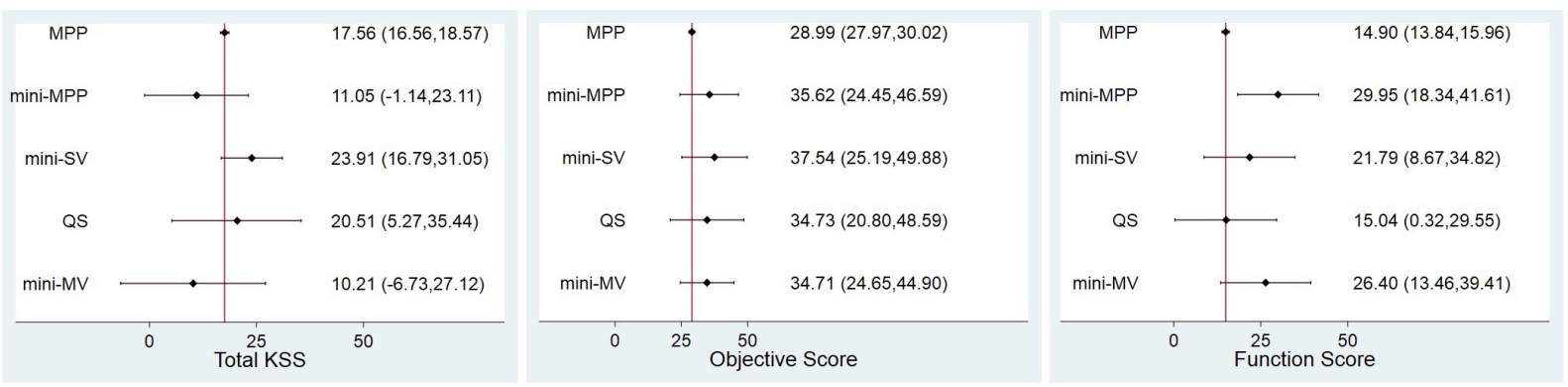

Fig. 4. Forest plot of network meta-analysis of all treatments for KSS improvement. MPP, medial parapatellar; MV, midvastus; QS, quadriceps-sparing; SV, subvastus.
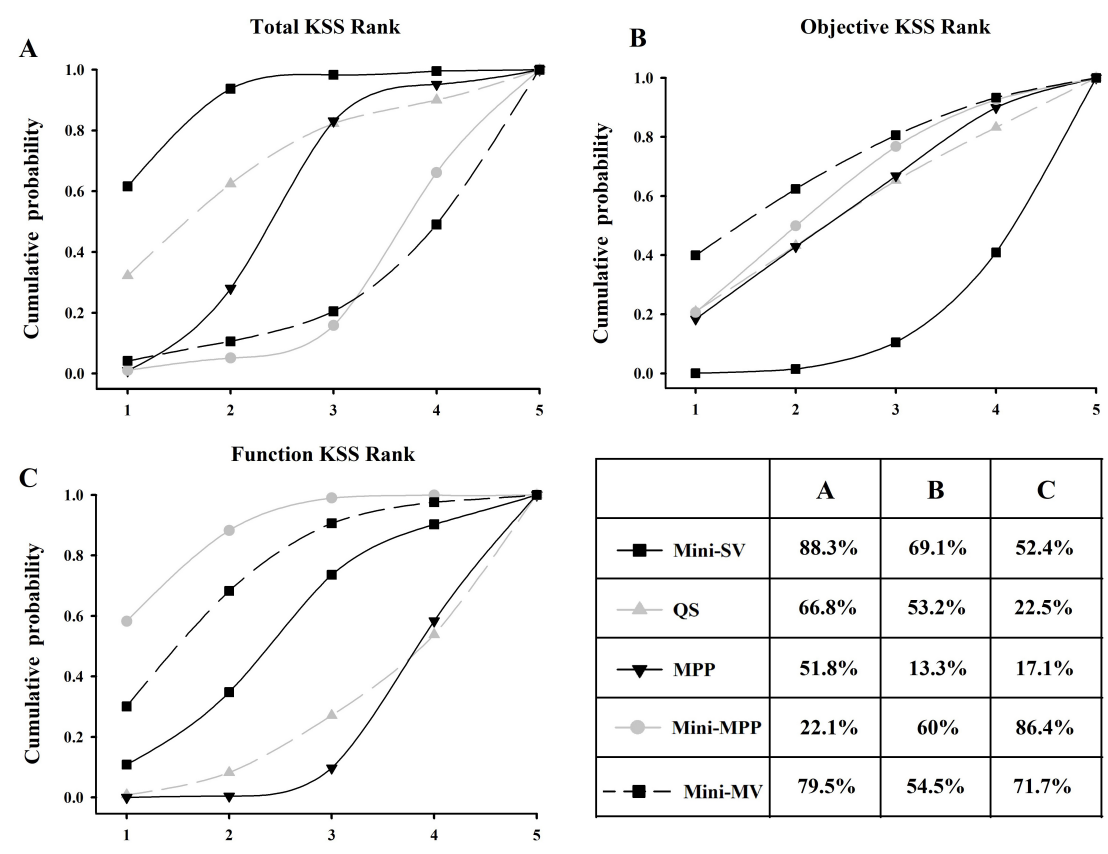

Fig. 5. Surface under the cumulative ranking curve (SUCRA) of the five approaches. The area under the curve represents the cumulative rank probability of each treatment. The larger the area, the better the cumulative rank probability. MPP, medial parapatellar; MV, midvastus; QS, quadriceps-sparing; SV, subvastus.

values. In terms of total KSS, mini-SV and QS approaches corresponded to greater but not significant improvement compared with standard MPP (MD $=-6.35,95 \%$ CrI: $(-13.39,0.67)$; MD $=-2.95,95 \%$ CrI: $(-17.92,12.21)$, respectivel)]. In contrast, mini-MPP and mini-MV showed less improvement (MD $=6.51,95 \%$ CrI: $(-5.46,18.07) ; \mathrm{MD}=7.35,95 \% \mathrm{CrI}:(-9.52,24.35)$, respectively). With regard to objective and functional score, there is a trend favoring MIS approaches over standard MPP approaches, but the only significant difference was found for mini-MPP (MD $=-15.05,95 \%$ CrI: $(-26.69,-3.45)$ ).

No statistically significant differences were observed when the total and objective knee score was compared between MIS groups at four-eight weeks. The only statistically significant differences were observed when the function score was compared between mini-MPP and QS (MD $=14.91,95 \% \mathrm{CrI}$ : $(0.86,29.04))$. 
Table 3

Results of inconsistency analysis and meta-regression

\begin{tabular}{|c|c|c|c|c|c|c|}
\hline & \multicolumn{2}{|c|}{ Total KSS } & \multicolumn{2}{|c|}{ Objective score } & \multicolumn{2}{|c|}{ Function score } \\
\hline & Comparison & $p$-value & Comparison & $p$-value & Comparison & $p$-value \\
\hline \multirow[t]{3}{*}{ Inconstency } & \multirow{3}{*}{\multicolumn{2}{|c|}{ NA }} & MPP vs mini-MPP & 0.94 & MPP vs mini-MPP & 0.99 \\
\hline & & & MPP vs QS & 0.94 & MPP vs QS & 0.99 \\
\hline & & & Mini-MPP vs QS & 0.94 & Mini-MPP vs QS & 0.99 \\
\hline \multirow[t]{2}{*}{ DIC } & $\begin{array}{l}\text { Without the } \\
\text { covariate }\end{array}$ & $\begin{array}{l}\text { With the } \\
\text { covariate }\end{array}$ & $\begin{array}{l}\text { Without the } \\
\text { covariate }\end{array}$ & $\begin{array}{l}\text { With the } \\
\text { covariate }\end{array}$ & $\begin{array}{l}\text { Without the } \\
\text { covariate }\end{array}$ & $\begin{array}{l}\text { With the } \\
\text { covariate }\end{array}$ \\
\hline & 48.87 & 48.82 & 86.57 & 86.71 & 76.89 & 76.88 \\
\hline The regression coefficient & \multicolumn{2}{|c|}{$0.09583(-0.1528,0.3558)$} & \multicolumn{2}{|c|}{$0.08272(0.0004225,0.1643)$} & \multicolumn{2}{|c|}{$0.1445(0.06048,0.2253)$} \\
\hline
\end{tabular}

MPP, medial parapatellar; MV, midvastus; QS, quadriceps-sparing; SV, subvastus.

\subsection{Forest plot of all treatments for KSS improvement}

The results of KSS improvement are presented in Fig. 4. All treatments showed statistically significant superiority when compared with pre-operative score except for mini-MPP and mini-MV with regard to total KSS scores.

\subsection{Rank probability based on SUCRA}

The ranking probability of each treatment in terms of KSS improvement is illustrated in Fig. 5. The area under the curve represents the cumulative rank probability of each treatment. Larger areas under the SUCRA curve represent better effectiveness. Mini-MV showed the highest probability of being the best treatment for improving total (79.5\%) and objective (81.1\%) KSS, respectively. Mini-MPP showed the highest probability $(86.4 \%)$ of being the best treatment for improving functional KSS, followed by mini-MV (71.7\%) and mini-SV (52.4\%). It also ranked the second in improving objective KSS. Standard MPP, however, showed the lowest probability of being the best approach to improve objective (13.3\%) and function $(17.1 \%) \mathrm{KSS}$.

\subsection{Inconsistency analysis}

In objective and function KSS, six comparisons were necessary to detect the inconsistency. All the results are listed in Table 3. $p>0.05$ demonstrates that no difference existed between the direct and indirect results of these comparisons. Thus, no inconsistency was detected.

\subsection{Sensitivity analysis and meta-regression}

A low-quality study (Thienpont 2012) included objective KSS and function KSS's data. The comparison in the study is mini-MPP vs. MPP. After excluding this pair of data, the rank probability of two networks was calculated again. After exclusion, in the objective result, mini-SV (from 69.1\% changed to 83.5\%) was still ranked as number one and was better than mini-MV (from 54.5\% changed to 72\%), QS (53.2\% to $40.7 \%$ ), MPP (13.3\% to $33.2 \%$ ), and mini-MPP (60.0\% to $20.6 \%)$. In function KSS's result, mini-MV changed to number one from $71.7 \%$ to $90.0 \%$. Mini-SV ranked number two (from $52.4 \%$ changed to $71.5 \%$ ), mini-MPP declined to number three (from $86.4 \%$ changed to $45.2 \%$ ), and QS was number four ( $22.5 \%$ changed to $7.6 \%)$.

Meta-regression was performed on the three different KSS' networks, and no significant change in the DIC was observed (Table 3). These data suggest that the covariate (the sample size of the study) was associated with the treatment effects in objective and function KSS. 


\section{Discussion}

The present network meta-analysis compared the effectiveness of MIS approaches (mini-MPP, miniMV, mini-SV, QS) and standard MPP approach for TKA in terms of KSS improvement at four-eight weeks. The network results indicated that all five approaches improved KSS outcomes compared with preoperative data. There is also a trend favoring MIS approaches in KSS score over standard MPP approach at four-eight weeks. Only mini-MPP showed a statistically significant difference when compared with standard MPP and QS for function KSS score. In terms of ranking probability, mini-SV has the highest probability of improving total and objective KSS (88.3\% and 69.1\%, respectively). Mini-MPP has the highest probability $(86.4 \%)$ for improving functional KSS, followed by mini-MV (71.7\%) and mini-SV (52.4\%). Standard MPP approach was the least effective for objective and functional KSS score, 13.3\% and $17.1 \%$, respectively.

TKA surgeries alleviate pain, restore function and improve patient's satisfaction as inflammatory tissue and damaged cartilage are removed and alignment of the lower limb is fixed. Thus, it is one of the most successful and effective surgical procedures for patients with severe osteoarthritis. In accordance, Fig. 4 showed significantly improved total, objective and functional KSS after all approaches when compared with baseline data.

It is feasible to presume that the MIS approach may reveal better results when compared with standard approaches due to their limited damage to the extensor muscles and quadriceps tendon. In recent years, after retrospectively or at best prospectively compared cohorts (age/sex matched), MIS approaches seemed to fail to confer a substantial postoperative advantage in regard to some early outcomes such as hospital stay, VAS, KSS and straight leg raise $[7,27,28]$. This was confirmed by several other recent RCTs [29,30] and RCT meta-analyses [6,15,31-33]. In our pooled analysis, only mini-MPP showed statistically significantly better KSS function score improvement at postoperative four-eight weeks (MD $=-15.05,95 \%$ CrI: $(-26.69,-3.45)$ ) (Table 2$)$. In a prospective, randomized, multicenter study, Kolisek found no difference between a mini-midvastus approach and a standard approach with regard to mean objective and function KSS at six and 12 weeks postoperatively [30]. Guy et al. randomized 80 patients and performed the mini-midvastus approach or standard MPP approach and found no statistically significant differences in total KSS at intervals up to one year (three weeks, six weeks, nine weeks, 12 weeks, six months and one year) [29]. Furthermore, both Smith, Alcelik and Gandhi reported no statistical significance in terms of objective and functional KSS between MIS TKA and the conventional approach $[6,15,31]$ in meta-analyses of RCTs. However, this should be interpreted with caution since all MIS surgeries were combined together, and therefore specific approaches were not determined. Later, better-performed meta-analyses showed no statistical advantage for mini-MV over standard MPP groups in terms of KSS score in postoperative six weeks or later [33,34]. Similar results were also found in other meta-analyses for QS and mini-SV at postoperative four-six weeks [32].

The failure of demonstrating significant superior KSS score improvement in the MIS group could be possibly attributed to the following reasons: 1 . There is a learning curve for surgeons undergoing MIS TKA. Lack of experience may result in poor clinical outcome, leading to underestimation of the MIS group effect $[35,36]$. 2. Routinely applied advanced anesthetics, pain management, rapid rehabilitation, and patient education protocols which may accelerate the recovery of patients who accept conventional TKA procedure and thus narrow the difference. 3. Recently, some attention also shifted from the putatively objective tests (e.g. gait speed and duration of surgery) to the ultimately objective quantitative parameters such as serum biomarkers of muscle damage and inflammation (e.g. serum creatine kinas and C-reactive protein) [37-39]. Surprisingly, no superiority over MPP or even more muscle damage was found in the MIS approach [37,39], suggesting MIS TKA is not less muscle sparing than the conventional approach. 
In contrast to our results, several studies have reported that MIS TKA is associated with a significant better short-term KSS improvement. It should be noted that those non-randomized data comparing the outcomes of two surgical techniques must be interpreted with caution because they have the potential of a selection bias that may confound the relationship between the intervention and the outcome. Lin et al. performed a meta-analysis that compared the conventional MPP with the MIS approach [40] and found superior outcomes in KSS (objective and total) in the MIS approach. However, their analysis combined different MIS procedures in one analysis, hampering its clinical interpretation. Also, they did not integrate data over time. Most importantly, the primary outcome of our present study is the KSS improvement, not the difference within pre- and post-operation data, respectively. In a meta-analysis of 14 RCTs, Wu et al. also reported that mini-SV provides greater total KSS than MPP $(P=0.007)$ but not functional KSS $(P=0.31)$ [41]. This difference results from the following reasons: 1 . We adopted a four-eight weeks timepoint for the present study whereas $\mathrm{Wu}$ et al. did not. 2 . There is a disagreement in the studies included in our respective meta-analyses: Wu et al. included Bridgman's work [42], a study which compared subvastus (rather than the mini-SV approach) with MPP. This demonstrated a violation to its exclusion criteria. Furthermore, we included Yao's work [43] according to our inclusion criteria. These deviations led to the inappropriate inclusion of one study that reported results in favor of mini-SV, and the exclusion of a study that reported results in favor of our results, biasing results in favor of mini-SV.

Until now, there are a few studies which compared MIS approaches. Although they showed significantly improvements in short-term clinical outcomes and expedite return of function, there seems no substantive difference between the MIS approaches for TKA. Results of participants undergoing mMV and mMPP approach TKA demonstrate similar results for short- and long-term results in clinical parameters (VAS, KSS, HSS, ROM), radiographic component positioning and complication [44-49]. Notably, Heekin [49] and Sun [48] reported significantly better quadricep strength in the mini-MV group as measured by Cybex testing and straight leg raise whereas Zhang [47] and Liu [44] did not. In terms of QS vs. mini-MPP, Hung et al. reported no significant difference in any of the clinical (VAS, KSS, ROM) and radiographic outcomes after a minimum of five years of follow-up [50,51]. Lin reported similar results with a shorter follow-up from postoperative 24 hours to two years [12]. Aglietti found no difference between mini-subvastus and QS approaches with regard to operation time, blood loss, complications, postoperative pain (day one), degrees flexion (one, three, six months) and radiographic data. However, active straight leg raise was achieved slightly quicker in the mini-subvastus group $(p=0.04)$ [52]. Mini-SV was also reported to have similar clinical results but lower pain score at four weeks when compared with mini-MV [10].

Mini-SV and QS avoid damaging the quadriceps tendon and muscle. Thus, it is reasonable to expect that such approaches could lead to superior functional improvement over other approaches. However, none of our results supported this. On the contrary, our pooled results revealed that mini-MPP better improved KSS function score over QS (MD $=14.91,95 \%$ CrI: $(0.86,29.04))$. This might be because mini-medial parapatellar is the most similar to the routine parapatellar approach. It is easy to master, quick to perform and potentially extendable. The QS and mini-SV are more technically demanding and a proper patient selection is required [53].

First, there appeared a number of methodological variations. For example, those studies with poorly concealing randomization of subjects would result in potential allocation bias. Also, although surgeons could not be blinded to the study allocation, further bias from patient expectation and the clinical outcome assessor might compromise the internal validity of a trial. Second, only a small number of studies based their sample size on a power calculation. As a result, such studies may lack sufficient power to demonstrate significant acceleration of clinical recovery after MIS approaches and are unable to detect 
a statistical difference between the two different procedures resulting in type II statistical error. Third, surgical experience could be an additional source of bias in this study and potential confound results [36]. A learning phase was identified using the MIS technique, as they are more technically demanding. A sudden jump from standard TKA approaches to MIS approaches will exposes patients to unnecessary risks and compromises surgical benefits. In the present study, only five included studies quantified the experience of participating surgeons [12,27,54-56]. Furthermore, even for the "experienced" surgeon who was assigned to perform MIS TKA to eliminate such bias, it cannot be presumed that the ability to perform the procedures in each arm of RCTs would be managed at the same standard and thus the intervention effect could be under- or over-estimated [12]. This may explain the heterogeneity.

Thus, with the early wide deviations of the "outcomes pendulum" logically coming to equilibrium, there is little left to extract from the continuation of traditional meticulous comparisons and debates over which approach is better. Perhaps future attention should focus on the clarification of more specific and strict indications and contraindications for each approach (e.g., comorbidities, degree of flexion/extension, angle of valgus/varus deformity, etc.). Moreover, improvement of surgical technique, modified implant design, intra-operative navigation system as well as postoperative rehabilitation should be advocated in both standard and MIS TKA procedures as they may help to achieve better postoperative function and better patient satisfaction.

\subsection{Advantages and strengths}

This study has some advantages and strengths. This is the first Bayesian network meta-analysis, which compares all available MIS TKA as well as conventional TKA. Unlike conventional metaanalyses techniques, Bayesian methodology advantageously enabled us to simultaneously compare every approach. We included the KSS score system as our primary outcome to assess patient outcomes, as it is a powerful scoring system with extensive validation. Our primary outcome is KSS improvement rather than postoperative KSS score. This study only included RCTs with a prospective design criterion. Furthermore, all p-values regarding the inconsistencies were less than 0.05 . The sensitivity analysis did not show a significant change in the cumulative probabilities rank, and no significant change in the DIC was found according to the meta-regression results. Therefore, the outcome of this meta-analysis is valid and reliable. In addition, we calculated indirect comparisons via Bayesian statistics. Furthermore, this model was used to build inconsistency, sensitivity and meta-regression tests.

\subsection{Study limitations}

We acknowledge some limitations in this study. First, due to the lack of available trial data, our study provides results only at postoperative four-eight weeks and thus we are unable to ascertain the longer-term comparison result between these five approaches. Also, the direct estimates for some procedures are based on a single study. Thus, we highlight the need for more high-quality prospective RCTs with greater sample sizes and longer follow-up periods in the future to supplement and corroborate these results.

Second, the qualities of the included studies varied. Some studies were better-designed RCTs with high patient numbers and adequate randomization; however, other studies had few participants or weak blinding/allocation. This limitation can be addressed after more high-quality studies are conducted in the future.

Third, heterogeneity exists within particular subgroups. Variations which may have accounted for such heterogeneity include: the difference in sample sizes, different inclusion and exclusion criteria, the variations in randomization and blinding, experience of MIS approaches, the use of a wide variety 
of implants and navigation systems, and variability in the use of minimally invasive techniques and postoperative pain management standardization. Unfortunately, not all studies quantified these factors and our review ignored these differences. This uncertainty is magnified when integrating them in mixedtreatment comparisons, as evidenced by the enlarged 95\% CIs. This may explain why most of our comparisons were statistically insignificant.

\section{Conclusion}

Based on the current evidence, the MIS approach was not significantly statistically superior to the standard parapatellar approach in terms of KSS scores at postoperative four-eight weeks. We recommend that the choice of the surgical approach is based on surgeon experience and preference as well as patients' particular characteristics and demands.

\section{Conflict of interest}

The authors declare that they have no conflict of interest.

\section{Supplementary data}

Appendices 1-3 are available to download from http://dx.doi.org/10.3233/THC-192078.

\section{References}

[1] Tsuji S, Tomita T, Fujii M, Laskin RS, Yoshikawa H, Sugamoto K. (2010). Is minimally invasive surgery-total knee arthroplasty truly less invasive than standard total knee arthroplasty? A quantitative evaluation. The Journal of Arthroplasty 25(6): 970-976.

[2] Shen H, Zhang X, Wang Q, Shao J, Jiang Y. (2007). Minimally invasive total knee arthroplasty through a quadriceps sparing approach: A comparative study. Zhonghua Wai Ke Za Zhi [Chinese Journal of Surgery] 45(16): 1083-1086.

[3] Tasker A, Hassaballa M, Murray J, Lancaster S, Artz N, Harries W, Porteous A. (2014). Minimally invasive total knee arthroplasty; a pragmatic randomised controlled trial reporting outcomes up to 2 year follow up. The Knee 21(1): 189-193.

[4] Cho K-Y, Kim K-I, Umrani S, Kim S-H. (2014). Better quadriceps recovery after minimally invasive total knee arthroplasty. Knee Surgery, Sports Traumatology, Arthroscopy 22(8): 1759-1764.

[5] Shin YS, Kim HJ, Ko YR, Yoon JR. (2016). Minimally invasive navigation-assisted versus conventional total knee arthroplasty: A meta-analysis. Knee Surgery, Sports Traumatology, Arthroscopy: Official Journal of the ESSKA 24(11): 3425-3432. doi: 10.1007/s00167-016-4016-2.

[6] Smith TO, King JJ, Hing CB. (2012). A meta-analysis of randomised controlled trials comparing the clinical and radiological outcomes following minimally invasive to conventional exposure for total knee arthroplasty. The Knee 19(1): 1-7. doi: 10.1016/j.knee.2010.12.001.

[7] Nestor BJ, Toulson CE, Backus SI, Lyman SL, Foote KL, Windsor RE. (2010). mini-midvastus vs standard medial parapatellar approach: A prospective, randomized, double-blinded study in patients undergoing bilateral total knee arthroplasty. Journal of Arthroplasty 25(6): 5-11.e11.

[8] Tashiro Y, Miura H, Matsuda S, Okazaki K, Iwamoto Y. (2007). Minimally invasive versus standard approach in total knee arthroplasty. Clinical Orthopaedics and Related Research (1976-2007) 463: 144-150.

[9] Dalury DF, Dennis DA. (2005). Mini-incision total knee arthroplasty can increase risk of component malalignment. Clinical Orthopaedics and Related Research ${ }^{\circledR}$ 440: 77-81.

[10] Bonutti PM, Zywiel MG, Ulrich SD, McGrath MS, Mont MA. (2010). Minimally invasive total knee arthroplasty: Pitfalls and complications. Am J Orthop (Belle Mead NJ) 39(10): 480-484. 
[11] Tzatzairis T, Fiska A, Ververidis A, Tilkeridis K, Kazakos K, Drosos GI. (2018). Minimally invasive versus conventional approaches in total knee replacement/arthroplasty: A review of the literature. J Orthop 15(2): 459-466. doi: 10.1016/j.jor.2018.03.026.

[12] Lin S, Chen C, Fu Y, Huang P, Lu C, Su J, Chang J, Huang H. (2013). Comparison of the clinical and radiological outcomes of three minimally invasive techniques for total knee replacement at two years. The Bone \& Joint Journal 95(7): 906-910.

[13] Aso K, Ikeuchi M, Izumi M, Kato T, Tani T. (2012). Transcutaneous oxygen tension in the anterior skin of the knee after minimal incision total knee arthroplasty. The Knee 19(5): 576-579.

[14] Yuan F-Z, Wang S-J, Zhou Z-X, Yu J-K, Jiang D. (2017). Malalignment and malposition of quadriceps-sparing approach in primary total knee arthroplasty: A systematic review and meta-analysis. Journal of Orthopaedic Surgery and Research 12(1): 129.

[15] Alcelik I, Sukeik M, Pollock R, Misra A, Shah P, Armstrong P, Dhebar MI. (2012). Comparison of the minimally invasive and standard medial parapatellar approaches for primary total knee arthroplasty. Knee Surgery, Sports Traumatology, Arthroscopy: Official Journal of the ESSKA 20(12): 2502-2512. doi: 10.1007/s00167-012-1944-3.

[16] Liberati A, Altman DG, Tetzlaff J, Mulrow C, Gøtzsche PC, Ioannidis JP, Clarke M, Devereaux PJ, Kleijnen J, Moher D. (2009). The PRISMA statement for reporting systematic reviews and meta-analyses of studies that evaluate health care interventions: Explanation and elaboration. PLoS Medicine 6(7): e1000100.

[17] Guyatt GH, Oxman AD, Vist GE, Kunz R, Falck-Ytter Y, Alonso-Coello P, Schünemann HJ. (2008). GRADE: An emerging consensus on rating quality of evidence and strength of recommendations. BMJ (Clinical Research Ed) 336(7650): 924-926.

[18] Salanti G, Del Giovane C, Chaimani A, Caldwell DM, Higgins JP. (2014). Evaluating the quality of evidence from a network meta-analysis. PloS One 9(7): e99682.

[19] Puhan MA, Schünemann HJ, Murad MH, Li T, Brignardello-Petersen R, Singh JA, Kessels AG, Guyatt GH. (2014). A GRADE working group approach for rating the quality of treatment effect estimates from network meta-analysis. Bmj 349: g5630.

[20] Jonas DE, Wilkins TM, Bangdiwala S, Bann CM, Morgan LC, Thaler KJ, Amick HR, Gartlehner G. (2013). AHRQ Methods for Effective Health Care. In: Findings of Bayesian Mixed Treatment Comparison Meta-Analyses: Comparison and Exploration Using Real-World Trial Data and Simulation. Agency for Healthcare Research and Quality (US), Rockville (MD).

[21] Brooks SP, Gelman A. (1998). General methods for monitoring convergence of iterative simulations. J Comput Graph Stat 7(4): 434-455. doi: 10.2307/1390675.

[22] Chaimani A, Higgins JP, Mavridis D, Spyridonos P, Salanti G. (2013). Graphical tools for network meta-analysis in STATA. PloS One 8(10): e76654. doi: 10.1371/journal.pone.0076654.

[23] Dias S, Welton NJ, Caldwell DM, Ades AE. (2010). Checking consistency in mixed treatment comparison meta-analysis. Statistics in Medicine 29(7-8): 932-944. doi: 10.1002/sim.3767.

[24] van Valkenhoef G, Dias S. (2016). Automated generation of node-splitting models for assessment of inconsistency in network meta-analysis. 7(1): 80-93. doi: 10.1002/jrsm.1167.

[25] Dias S, Sutton AJ, Welton NJ, Ades AE. (2012). NICE Decision Support Unit Technical Support Documents. In: Heterogeneity: Subgroups, Meta-Regression, Bias And Bias-Adjustment. NICE Decision Support Unit Technical Support Documents. National Institute for Health and Care Excellence (NICE) unless otherwise stated. All rights reserved., London.

[26] Spiegelhalter DJ, Best NG, Carlin BP, Linde AVD. (2002). Bayesian measures of model complexity and fit. J R Stat Soc Ser B Stat Methodol. Journal of the Royal Statistical Society 64(4): 583-639.

[27] Wegrzyn J, Parratte S, Coleman-Wood K, Kaufman KR, Pagnano MW. (2013). The John Insall award: No benefit of minimally invasive TKA on gait and strength outcomes: A randomized controlled trial. Clinical Orthopaedics and Related Research $^{\circledR}$ 471(1): 46-55.

[28] Tomek IM, Kantor SR, Cori LA, Scoville JM, Grove MR, Morgan TS, Swarup I, Moschetti WE, Spratt KF. (2014). Early patient outcomes after primary total knee arthroplasty with quadriceps-sparing subvastus and medial parapatellar techniques: A randomized, double-blind clinical trial. The Journal of Bone and Joint Surgery American Volume 96(11): 907.

[29] Guy SP, Farndon MA, Conroy JL, Bennett C, Grainger AJ, London NJ. (2012). A prospective randomised study of minimally invasive midvastus total knee arthroplasty compared with standard total knee arthroplasty. The Knee 19(6): 866-871. doi: 10.1016/j.knee.2012.04.009.

[30] Kolisek FR, Bonutti PM, Hozack WJ, Purtill J, Sharkey PF, Zelicof SB, Ragland PS, Kester M, Mont MA, Rothman RH. (2007). Clinical experience using a minimally invasive surgical approach for total knee arthroplasty: Early results of a prospective randomized study compared to a standard approach. The Journal of Arthroplasty 22(1): 8-13. 
[31] Gandhi R, Smith H, Lefaivre KA, Davey JR, Mahomed NN. (2011). Complications after minimally invasive total knee arthroplasty as compared with traditional incision techniques: A meta-analysis. The Journal of Arthroplasty 26(1): 29-35. doi: 10.1016/j.arth.2009.11.022.

[32] Peng X, Zhang X, Cheng T, Cheng M, Wang J. (2015). Comparison of the quadriceps-sparing and subvastus approaches versus the standard parapatellar approach in total knee arthroplasty: A meta-analysis of randomized controlled trials. BMC Musculoskeletal Disorders 16: 327. doi: 10.1186/s12891-015-0783-z.

[33] Xu SZ, Lin XJ, Tong X, Wang XW. (2014). Minimally invasive midvastus versus standard parapatellar approach in total knee arthroplasty: A meta-analysis of randomized controlled trials. PloS One 9(5): e95311. doi: 10.1371/journal. pone. 0095311.

[34] Verburg H, Mathijssen NM, Niesten D-D, Verhaar JA, Pilot P. (2016). Comparison of mini-midvastus and conventional total knee arthroplasty with clinical and radiographic evaluation: A prospective randomized clinical trial with 5-year follow-up. JBJS 98(12): 1014-1022.

[35] Cheng YC, Wu PK, Chen CF, Chen CM, Tsai SW, Chang MC, Chen WM. (2019). Analysis of learning curve of minimally invasive total knee arthroplasty: A single surgeon's experience with 4017 cases over a nine-years period. Journal of the Chinese Medical Association: JCMA. doi: 10.1097/JCMA.0000000000000118.

[36] Carroll C, Mahmood F. (2019). Risk of bias and the reporting of surgeons' experience in randomized controlled trials of total hip and total knee arthroplasty: A systematic review. J Eval Clin Pract 25(2): 205-215. doi: 10.1111/jep.13056.

[37] Huang Z, Shen B, Ma J, Yang J, Zhou Z, Kang P, Pei F. (2012). Mini-midvastus versus medial parapatellar approach in TKA: Muscle damage and inflammation markers. Orthopedics 35(7): e1038-1045. doi: 10.3928/01477447-20120621-17.

[38] Yombi JC, Schwab PE, Thienpont E. (2015). Serum C-reactive protein distribution in minimally invasive total knee arthroplasty do not differ with distribution in conventional total knee arthroplasty. PloS One 10(4): e0124788. doi: 10.1371/journal.pone.0124788.

[39] Niki Y, Mochizuki T, Momohara S, Saito S, Toyama Y, Matsumoto H. (2009). Is minimally invasive surgery in total knee arthroplasty really minimally invasive surgery? The Journal of Arthroplasty 24(4): 499-504.

[40] Li C, Zeng Y, Shen B, Kang P, Yang J, Zhou Z, Pei F. (2015). A meta-analysis of minimally invasive and conventional medial parapatella approaches for primary total knee arthroplasty. Knee Surgery, Sports Traumatology, Arthroscopy 23(7): 1971-1985.

[41] Wu Y, Zeng Y, Bao X, Xiong H, Hu Q, Li M, Shen B. (2018). Comparison of mini-subvastus approach versus medial parapatellar approach in primary total knee arthroplasty: A meta-analysis of 14 randomized controlled trials. International journal of surgery.

[42] Bridgman SA, Walley G, Mackenzie G, Clement D, Griffiths D, Maffulli N. (2009). Sub-vastus approach is more effective than a medial parapatellar approach in primary total knee arthroplasty: A randomized controlled trial. The Knee 16(3): 216-222.

[43] Yao Y, Kang P, Xue C, Jing J. (2018). A prospective randomized controlled study of total knee arthroplasty via minisubvastus and conventional approach. Zhongguo xiu fu chong jian wai ke za zhi = Zhongguo xiufu chongjian waike zazhi $=$ Chinese journal of reparative and reconstructive surgery 32(2): 162-168.

[44] Liu H, Mei X, Zhang Z, Sun J. (2015). Mini-midvastus versus mini-medial parapatellar approach in simultaneous bilateral total knee arthroplasty with 24-month follow-up. Acta Orthop Traumatol Turc 49(6): 586-592.

[45] Pongcharoen B, Yakampor T, Charoencholvanish K. (2013). Patellar tracking and anterior knee pain are similar after medial parapatellar and midvastus approaches in minimally invasive TKA. Clinical Orthopaedics and Related Research ${ }^{\circledR}$ 471(5): 1654-1660.

[46] Lee D-H, Choi J, Nha K-W, Kim H-J, Han S-B. (2011). No difference in early functional outcomes for mini-midvastus and limited medial parapatellar approaches in navigation-assisted total knee arthroplasty: A prospective randomized clinical trial. Knee Surgery, Sports Traumatology, Arthroscopy 19(1): 66-73.

[47] Zhang Z, Zhu W, Gu B, Zhu L, Chen C. (2013). Mini-midvastus versus mini-medial parapatellar approach in total knee arthroplasty: A prospective, randomized study. Archives of Orthopaedic and Trauma Surgery 133(3): 389-395.

[48] Sun JY, Wang Q, Liang-Bo MA, Yin H. (2008). Compared the mini-midvastus with mini-paramedian approach for the total knee arthroplasty. Chinese Journal of Joint Surgery 2(3): 28-31.

[49] Heekin RD, Fokin AA. (2014). Mini-midvastus versus mini-medial parapatellar approach for minimally invasive total knee arthroplasty: Outcomes pendulum is at equilibrium. The Journal of Arthroplasty 29(2): 339-342.

[50] Huang A-B, Wang H-J, Yu J-K, Yang B, Ma D, Zhang J-Y. (2016). Optimal patellar alignment with minimally invasive approaches in total knee arthroplasty after a minimum five year follow-up. International Orthopaedics 40(3): 487-492.

[51] Huang A-B, Wang H-J, Yu J-K, Yang B, Ma D, Zhang J-Y. (2015). Are there any clinical and radiographic differences between quadriceps-sparing and mini-medial parapatellar approaches in total knee arthroplasty after a minimum 5 years of follow-up? Chinese Medical Journal 128(14): 1898.

[52] Aglietti P, Baldini A, Sensi L. (2006). Quadriceps-sparing versus mini-subvastus approach in total knee arthroplasty. Clinical Orthopaedics and Related Research ${ }^{\circledR}$ 452: 106-111. 
[53] Tria AJ, Scuderi GR. (2015). Minimally invasive knee arthroplasty: An overview. World Journal of Orthopedics 6(10): 804.

[54] Juosponis R, Tarasevicius S, Smailys A, Kalesinskas RJ. (2009). Functional and radiological outcome after total knee replacement performed with mini-midvastus or conventional arthrotomy: Controlled randomised trial. International Orthopaedics 33(5): 1233-1237.

[55] Lüring C, Beckmann J, Haiböck P, Perlick L, Grifka J, Tingart M. (2008). Minimal invasive and computer assisted total knee replacement compared with the conventional technique: A prospective, randomised trial. Knee Surgery, Sports Traumatology, Arthroscopy 16(10): 928-934.

[56] Varela-Egocheaga JR, Suárez-Suárez MA, Fernández-Villán M, González-Sastre V, Varela-Gómez JR, RodríguezMerchán C. (2010). Minimally invasive subvastus approach: improving the results of total knee arthroplasty: A prospective, randomized trial. Clinical Orthopaedics and Related Research ${ }^{\circledR}$ 468(5): 1200-1208. 